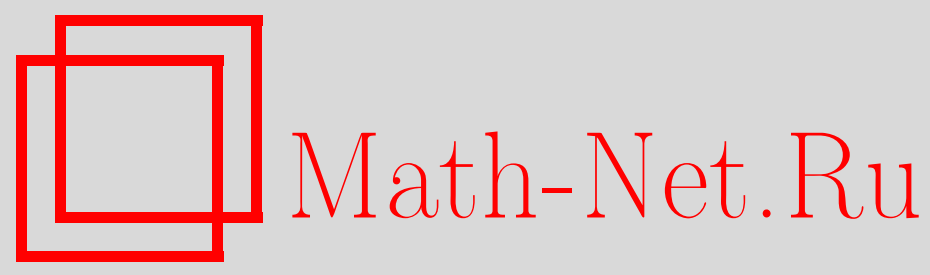

В. В. Тен, Топологические препятствия к интегрируемости избыточным набором интегралов, Матем. заметки, 1997, том 62, выпуск 5, 798-800

DOI: https://doi.org/10.4213/mzm1668

Использование Общероссийского математического портала Math-Net.Ru подразумевает, что вы прочитали и согласны с пользовательским соглашением http://www . mathnet.ru/rus/agreement

Параметры загрузки:

IP : 54.197 .217 .227

26 апреля 2023 г., $11: 15: 35$

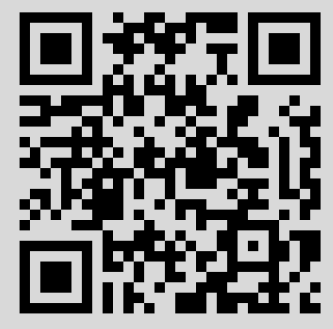




\section{ТОПОЛОГИЧЕСКИЕ ПРЕПЯТСТВИЯ К ИНТЕГРИРУЕМОСТИ ИЗБЫТОЧНЫМ НАБОРОМ ИНТЕГРАЛОВ}

\section{B. В. Тен}

1. Введение. Рассмотрим компактное аналитическое многообразие (далее все объекты аналитические) $M^{n}$ с римановой метрикой $g_{i j}$.

Риманова структура задает на фазовом пространстве $T^{*} M^{n}$ гамильтонову динамическую систему с гамильтонианом $H=\frac{1}{2} \sum g_{i j} p^{i} p^{j}$, которая называется геодезическим потоком. В силу траекторной эквивалентности потока на разных уровнях энергии $\{H=$ const $\}$ можно рассмотреть только один уровень $\{H=1\}$.

В дальнейшем нам понадобится следующее

ОПРЕДЕЛЕНИЕ. Геодезический поток назьвается геометрически простым, если единичное кокасательное расслоение $P=T^{*} M_{1}^{n}$ представляется в виде конечного объединения

$$
P=\bigsqcup_{i=1}^{s} B_{i} \sqcup \Gamma,
$$

где $B_{i}$ - инвариантные непересекающиеся диффеоморфные друг другу подмножества, а $\Gamma$ - замкнутое инвариантное подмножество.

Понятие геометрической простоты в более сильном смысле было введено в [1]. Можно показать, что в случае аналитической интегрируемости система всегда будет геометрически простой. При этом множество Г содержит множество особых точек набора первых интегралов.

Для доказательства используется метод, примененньй И. А. Таймановым в работе [1]. Изучаются топологические препятствия к интегрированию избыточным набором интегралов. Результат получен в предположении, что почти все совместные уровни интегралов - торы. Это верно, например, в случае некоммутативной интегрируемости [2].

2. Основной результат. Ниже формулируется теорема, содержащая основной результат работы.

ТЕОремА. Пусть геометрически простой геодезический поток имеет $n+k-1$ независимых дополнительных интегралов, так что $B_{i} \simeq \mathbb{T}^{n-k} \times D^{n+k-1}$. Тогда первое число Бетти $b_{1}\left(M^{n}\right) \leqslant n-k$.

Если $k=0$, то имеем условие полной интегрируемости. При $k=0$ и $n=2$ неравенство $b_{1}\left(M^{n}\right) \leqslant n$ получено В. В. Козловым в [3], а для $n>2$ И. А. Таймановьг [1].

В случае $n=2$ из теоремы вытекает интересное

СлЕдСТВИЕ. Если геодезический поток двумерного ориентируемого компактного многообразия имеет два независимых дополнительных интеграла, то это многообразие диффеоморфно сфере.

При $k=n-2$ получим, что $b_{1}\left(M^{n}\right) \leqslant 1$. Можно показать, что в этом случае все траектории замкнуты. При сопоставлении с результатами, описанными Бессе [4], мы

Работа выполнена при финансовой поддержке Российского фонда фундаментальных исследований, грант № 96-01-00747. 
видим очевидное их соответствие, хотя они гораздо сильнее в силу специальности примененного там апшарата.

3. Доказательство основного результата. Опишем алгебраическую конструкцию, которая нам понадобиться при доказательстве теоремы. Ее смысл станет понятен ниже. Рассмотрим тройку $(A, G, \mu)$, где $A$ - произвольное множество, $G$ - группа, а $\mu: G \rightarrow A \times A-$ произвольное отображение.

Построим ориентированньй граф $\Pi_{0}$, вершинами которого будут элементы $A$, а ребро $\left(a_{i}, a_{j}\right)$ появляется в случае, если эта пара служит прообразом некоторого элемента $G$. Естественно определяется отображение $\sigma$ из множества ребер графа $\Pi_{0}$ в групу $G$ (это отображение, обратное к $\mu$ ). Таким образом, мы пометили все ребра $\Pi_{0}$.

Достроим $\Pi_{0}$ до графа П следуюшим образом: если существует реброс меткой $g \in G$, то добавим ребро с обратной ориентацией и меткой $g^{-1}$.

Рассмотрим на графе П множество петель $\Omega$. Петлей назовем согласованньй с ориентацией путь $\gamma=\xi_{1} \ldots \xi_{m}$, где $\xi_{i}-$ ребра П, имеющий начало и конец в одной и той же вершине $a_{i} \in A$. Семейство петель, выходящих из $a_{i}$, обозначим через $\Omega_{i}$. На множестве петель можно определить отображение $\sigma_{1}$ так, что $\sigma_{1}(\gamma)=\sigma\left(\xi_{1}\right) \ldots \sigma\left(\xi_{m}\right)$. Очевидно, $\sigma_{1}\left(\Omega_{i}\right)=H_{i}$ является подгруппой $G$.

Сформулируем несколько лемм.

Лемма 1. Группа $G$ представляется в виде обвединения конечного числа левых смежных классов по подгруппам $H_{i}$.

Лемма 2. Пусть группа $G$ представляется в виде обвединения конечного числа левых смехных классов по подгруппам $H_{i}$. Тогда среди них найдется подгруппа конечного индекса.

Перейдем к приложению изложенной конструкции, к доказательству основной теоремы.

Рассмотрим некоторую достаточно малую окрестность $V$ произвольной точки $x_{0} \in$ $M^{n}$ конфигурационного многообразия. В силу определения геометрической простоты множество $L=\mathrm{pr}^{-1} V \backslash \Gamma$ является объединением конечного числа непересекающихся связных компонент $C_{1}, \ldots, C_{r}$. Выберем в каждой компоненте произвольную точку $q_{i}$.

По теореме Хопфа-Ринова, примененной к универсальной накрьвающей $M^{n}$, любой элемент фундаментальной групш $\pi_{1}\left(M^{n}\right)$ может быть реализован геодезической петлей с вершиной в одной точке $x_{0}$.

Поднимем эти петли в единичное кокасательное расслоение. Тогда их начала и концы будут лежать в прообразе $V$. Если некоторьй путь лежит в $\Gamma$, то как угодно мальп шевелением координат и импульсов он оттуда снимается.

Таким образом, можно построить множество путей, соединяющих $q_{i}$, которые при естественной проекции реализуют все элементы групшы $G$ (в $C_{i}$ конец пути соединяется с $q_{i}$ произвольным отрезком, лежашим внутри).

Теперь положим $A=\left\{q_{1}, \ldots, q_{r}\right\}, G=\pi_{1}\left(M^{n}\right)$, отображение $\mu$ индуцируется отображением, обратным к проекции, примененным к реализациям элементов фундаментальной группы. В силу инвариантности $B_{i}$ пути, соответствующие $\Omega_{j}(j=1, \ldots, r)$, содержатся в фундаментальной групе $\pi_{1}\left(B_{i}\right)$. По условию теоремы фундаментальная группа $\pi_{1}\left(B_{i}\right)=\pi_{1}\left(\mathbb{T}^{n-k}\right)$. Среди таких групп найдется подгрупша конечного индекса в $\pi_{1}\left(M^{n}\right)$, обозначим соответствующую связную компоненту через $B_{0}$. 
При помощи гомоморфизма Гуревича можно перейти к рассмотрению первых гомологий вместо фундаментальных групп. Рассмотрим первую группу гомологий $H_{1}\left(B_{0}\right)$. Так как она имеет конечньй индекс, проекция $\mathrm{pr}_{*}: H_{1}\left(B_{0}\right) \rightarrow H_{1}\left(M^{n}\right)$ является мономорфизмом, a pr* $H^{1}\left(M^{n}\right) \rightarrow H^{1}\left(B_{0}\right)$ - эпиморфизмом. Из последнего следует утверждение теоремы.

Автор благодарит В.В. Козлова за постановку задачи и внимание к работе.

\section{СПИСОК ЦИТИРОВАННОЙ ЛИТЕРАТУРЫ}

1. Тайманов И. А. // Матем. заметки. 1988. Т. 44. №2. С. 283-284. 2. Мищенко А. С., Фоменко А. Т. // Функцион. анализ и его прилож. 1987. Т. 12. № 2. С. 46-56. 3. Козлов В. В. // Докл. АН СССР. 1979. Т. 249. №6. С. 1299-1302. 4. Бессе А. Многообразия с замкнутыми геодезическими. М.: Мир, 1981.

Московский государственный университет

E-mail: vvten@nw.math.msu.su 